\title{
On the global existence of 3-D
} magneto-hydrodynamic system in the critical spaces

\section{Xiaolian $\mathrm{Ai}^{1}$ and Zilai $\mathrm{Li}^{2^{*}}$}

\section{"Correspondence:} lizilai0917@163.com

25chool of Mathematics and Information Science, Henan Polytechnic University, Jiaozuo, 454000, P.R. China

Full list of author information is available at the end of the article

\begin{abstract}
In this article, we prove the global existence of the three-dimensional inhomogeneous incompressible magneto-hydrodynamic system under the assumptions that the initial velocity field and the initial conductivity are small in the critical space $\dot{B}_{2,1}^{1 / 2}\left(\mathbb{R}^{3}\right)$.
\end{abstract}

MSC: 35Q35; 76D03

Keywords: magneto-hydrodynamic system; Littlewood-Paley theory; critical spaces

\section{Introduction}

In this paper, we consider the three-dimensional inhomogeneous incompressible magneto-hydrodynamics (MHD) which describes the coupling between the inhomogeneous Navier-Stokes system and the Maxwell equation [1]. We have

$$
\left\{\begin{array}{l}
\partial_{t} \rho+\operatorname{div}(\rho u)=0, \quad(t, x) \in \mathbb{R}^{+} \times \mathbb{R}^{3}, \\
\partial_{t}(\rho u)+\operatorname{div}(\rho u \otimes u)-\operatorname{div}(\mu \mathcal{M})+\nabla \Pi=B \cdot \nabla B, \\
\partial_{t} B+u \cdot \nabla B-\Delta B=B \cdot \nabla u, \\
\operatorname{div} u=0, \quad \operatorname{div} B=0, \\
\left.\rho\right|_{t=0}=\rho_{0},\left.\quad \rho u\right|_{t=0}=m_{0},\left.\quad B\right|_{t=0}=B_{0},
\end{array}\right.
$$

where the unknowns are the density $\rho$, the velocity $u=\left(u_{1}, u_{2}, u_{3}\right)$, the magnetic field $B=$ $\left(B_{1}, B_{2}, B_{3}\right)$, and the pressure function $\Pi . \mathcal{M}=\frac{1}{2}\left(\partial_{i} u_{j}+\partial_{j} u_{i}\right)$ is the deformation tensor and, in general, the viscosity coefficient $\mu$ is a smooth, positive function of the density $\rho$.

For the inhomogeneous MHD system (1.1), many results have been obtained. Gerbeau and Le Bris [2, 3], obtained global existence of weak solutions in whole space $\mathbb{R}^{3}$ or in the torus $T^{3}$. Abidi and Hmidi [4] proved the global existence of strong solutions with small initial density in Besov spaces. Moreover, Abidi and Hmidi [4] allowed for variable viscosity and conductivity coefficients but made the essential assumption that the initial data are closed to a constant state. Chen et al. [5] established the local strong solution in the presence of vacuum under the assumptions that both conductivity and viscosity are constants. The global existence of strong solutions was obtained by Huang and Wang [6]. Recently, Gui [7] showed global well-posedness of the two-dimensional inhomogeneous

(c) $2015 \mathrm{Ai}$ and $\mathrm{Li}$. This article is distributed under the terms of the Creative Commons Attribution 4.0 International License (http://creativecommons.org/licenses/by/4.0/), which permits unrestricted use, distribution, and reproduction in any medium, provided you give appropriate credit to the original author(s) and the source, provide a link to the Creative Commons license, and indicate if changes were made. 
MHD system with a constant viscosity and variable conductivity coefficients, but without the small density assumption.

If there is no magnetic field $(B=0)$, the MHD system turns out to be the inhomogeneous Navier-Stokes equations. In fact, due to the similarity of the second equation and the third equation in (1.1), the study of the MHD system has been along with that for NavierStokes one. There are a lot of studies of incompressible inhomogeneous Navier-Stokes equations. We should mention that Abidi et al. [8] proved the local well-posedness of the three-dimensional inhomogeneous incompressible isentropic Navier-Stokes equations in critical spaces but without the small density assumption. Motivated by [8], we shall investigate the global well-posedness of the 3-D incompressible inhomogeneous MHD (1.1) with constant viscosity coefficient in the critical spaces.

If the density $\rho$ is away from zero, we denote $a \stackrel{\text { def }}{=} \rho^{-1}-1$ and $\tilde{\mu}(a) \stackrel{\text { def }}{=} \mu(\rho)$, then the system (1.1) can be equivalently reformulated as

$$
\left\{\begin{array}{l}
\partial_{t} a+u \cdot \nabla a=0, \quad(t, x) \in \mathbb{R}^{+} \times \mathbb{R}^{N}, \\
\partial_{t} u+u \cdot \nabla u+(1+a)(\nabla \Pi-\operatorname{div}(\tilde{\mu}(a) \mathcal{M}))=(1+a) B \cdot \nabla B, \\
\partial_{t} B+u \cdot \nabla B-\Delta B=B \cdot \nabla u, \\
\operatorname{div} u=0, \quad \operatorname{div} B=0, \\
\left.(a, u, B)\right|_{t=0}=\left(a_{0}, u_{0}, B_{0}\right) .
\end{array}\right.
$$

For simplicity, we just take $\mu(\rho)=1$ and the space dimension $N=3$. In this case, (MHD) becomes

$$
\left\{\begin{array}{l}
\partial_{t} a+u \cdot \nabla a=0, \quad(t, x) \in \mathbb{R}^{+} \times \mathbb{R}^{3} \\
\partial_{t} u+u \cdot \nabla u+(1+a)(\nabla \Pi-\Delta u)=(1+a) B \cdot \nabla B \\
\partial_{t} B+u \cdot \nabla B-\Delta B=B \cdot \nabla u \\
\operatorname{div} u=0, \quad \operatorname{div} B=0 \\
\left.(a, u, B)\right|_{t=0}=\left(a_{0}, u_{0}, B_{0}\right)
\end{array}\right.
$$

or equivalently

$$
\left\{\begin{array}{l}
\partial_{t} \rho+u \cdot \nabla \rho=0, \quad(t, x) \in \mathbb{R}^{+} \times \mathbb{R}^{3}, \\
\partial_{t}(\rho u)+\operatorname{div}(\rho u \otimes u)-\Delta u+\nabla \Pi=B \cdot \nabla B \\
\partial_{t} B+u \cdot \nabla B-\Delta B=B \cdot \nabla u \\
\operatorname{div} u=0, \quad \operatorname{div} B=0 \\
\left.(a, u, B)\right|_{t=0}=\left(a_{0}, u_{0}, B_{0}\right) .
\end{array}\right.
$$

Our main result in this paper is as follows.

Theorem 1.1 Let $a_{0} \in B_{2,1}^{3 / 2}\left(\mathbb{R}^{3}\right), u_{0} \in \dot{B}_{2,1}^{1 / 2}\left(\mathbb{R}^{3}\right)$, and $B_{0} \in \dot{B}_{2,1}^{1 / 2}\left(\mathbb{R}^{3}\right)$ with $\operatorname{div} u_{0}=0$, $\operatorname{div} B_{0}=$ 0 , and

$$
1+a_{0} \geq \underline{b}
$$

for some positive constant $\underline{b}$. There exists a small constant $c$ depending on $\left\|a_{0}\right\|_{B_{2,1}^{3 / 2}}$ so that if

$$
\left\|u_{0}\right\|_{\dot{B}_{2,1}^{1 / 2}}+\left\|B_{0}\right\|_{\dot{B}_{2,1}^{1 / 2}} \leq c
$$


then (1.2) has a unique global solution $(a, u, B)$ satisfying

$$
\begin{aligned}
& \|a\|_{\tilde{L}_{t}^{\infty}\left(B_{2,1}^{3 / 2}\right)}+\|(u, B)\|_{\tilde{L}_{t}^{\infty}\left(\dot{B}_{2,1}^{1 / 2}\right)}+\|(u, B)\|_{L_{t}^{1}\left(\dot{B}_{2,1}^{5 / 2}\right)}+\|\nabla \Pi\|_{L_{t}^{1}\left(\dot{B}_{2,1}^{1 / 2}\right)} \\
& \quad \leq C\left(\left\|a_{0}\right\|_{B_{2,1}^{3 / 2}}+\left\|u_{0}\right\|_{\dot{B}_{2,1}^{1 / 2}}+\left\|B_{0}\right\|_{\dot{B}_{2,1}^{1 / 2}}+1\right) \exp \{C \sqrt{t}\} \quad \text { for any } t>0 .
\end{aligned}
$$

We now make some comments on the analysis of this paper. Motivated by [8], in order to prove the global well-posedness of Theorem 1.1, we note that as long as $\left\|u_{0}\right\|_{\dot{B}_{2,1}^{1 / 2}}+\left\|B_{0}\right\|_{\dot{B}_{2,1}^{1 / 2}}$ is sufficiently small, the lifespan of the local solution thus obtained should be greater than 1 , moreover, there exists $t_{1} \in(0,1)$ so that

$$
\left\|u\left(t_{1}\right)\right\|_{\dot{B}_{2,1}^{1 / 2} \cap \dot{B}_{2,1}^{7 / 2}}+\left\|B\left(t_{1}\right)\right\|_{\dot{B}_{2,1}^{1 / 2} \cap \dot{B}_{2,1}^{7 / 2}} \leq C\left(\left\|u_{0}\right\|_{\dot{B}_{2,1}^{1 / 2}}+\left\|B_{0}\right\|_{\dot{B}_{2,1}^{1 / 2}}\right)
$$

We shall first solve $v$ via the classical Navier-Stokes system:

$$
\left\{\begin{array}{l}
\partial_{t} v+v \cdot \nabla v-\Delta v+\nabla \Pi_{v}=0 \\
\operatorname{div} v=0 \\
\left.v\right|_{t=t_{1}}=u\left(t_{1}\right)
\end{array}\right.
$$

and then solve $w=u-v$ via

$$
\left\{\begin{array}{l}
\partial_{t} \rho+\operatorname{div}(\rho(v+w))=0 \\
\rho \partial_{t} w+\rho(v+w) \cdot \nabla w-\Delta w+\nabla \Pi_{w}=(1-\rho)\left(\partial_{t} v+v \cdot \nabla v\right)-\rho w \cdot \nabla v+B \cdot \nabla B \\
\partial_{t} B+(v+w) \cdot \nabla B-\Delta B=B \cdot \nabla(v+w) \\
\operatorname{div} w=0, \quad \operatorname{div} B=0, \\
\left.\rho\right|_{t=t_{1}}=\rho\left(t_{1}\right),\left.\quad w\right|_{t=t_{1}}=0,\left.\quad B\right|_{t=t_{1}}=B\left(t_{1}\right) .
\end{array}\right.
$$

The rest of the paper is organized as follows. In Section 2, we collect some elementary facts on Littlewood-Paley analysis that will be used later; then in Section 3, based on the local existence of the solutions and the priori estimates, we prove Theorem 1.1 by a standard continuity argument.

\section{Preliminaries}

Let us briefly explain how we may proceed in the case $x \in \mathbb{R}^{3}$ (see e.g. [9]). Let $\varphi$ be a smooth function supported in the $\operatorname{ring} \mathcal{C} \stackrel{\text { def }}{=}\left\{\xi \in \mathbb{R}^{3}, \frac{3}{4} \leq|\xi| \leq \frac{8}{3}\right\}$ and such that

$$
\sum_{q \in \mathbb{Z}} \varphi\left(2^{-q} \xi\right)=1 \quad \text { for } \xi \neq 0
$$

Then, for $u \in \mathcal{S}^{\prime}\left(\mathbb{R}^{3}\right)$, we set

$$
\forall q \in \mathbb{Z}, \quad \dot{\Delta}_{q} u=\varphi\left(2^{-q} \mathrm{D}\right) u \quad \text { and } \quad \dot{S}_{q} u=\sum_{j \leq q-1} \dot{\Delta}_{j} u .
$$

We have the formal decomposition

$$
u=\sum_{q \in \mathbb{Z}} \dot{\Delta}_{q} u, \quad \forall u \in \mathcal{S}^{\prime}\left(\mathbb{R}^{3}\right) / \mathcal{P}\left[\mathbb{R}^{3}\right]
$$


where $\mathcal{P}\left[\mathbb{R}^{3}\right]$ is the set of polynomials (see [10]). Moreover, the Littlewood-Paley decomposition satisfies the property of almost orthogonality:

$$
\dot{\Delta}_{k} \dot{\Delta}_{q} u \equiv 0 \quad \text { if }|k-q| \geq 2 \quad \text { and } \quad \dot{\Delta}_{k}\left(\dot{S}_{q-1} u \dot{\Delta}_{q} u\right) \equiv 0 \quad \text { if }|k-q| \geq 5
$$

We recall now the definition of the homogeneous Besov spaces from [11].

Definition 2.1 Let $(p, r) \in[1,+\infty]^{2}, s \in \mathbb{R}$, and $u \in \mathcal{S}^{\prime}\left(\mathbb{R}^{3}\right)$, we set

$$
\|u\|_{\dot{B}_{p, r}^{s}} \stackrel{\text { def }}{=}\left(2^{q s}\left\|\dot{\Delta}_{q} u\right\|_{L^{p}}\right)_{\ell^{r}}
$$

- For $s<\frac{3}{p}$ (or $s=\frac{3}{p}$ if $r=1$ ), we define $\dot{B}_{p, r}^{s}\left(\mathbb{R}^{3}\right) \stackrel{\text { def }}{=}\left\{u \in \mathcal{S}^{\prime}\left(\mathbb{R}^{3}\right) \mid\|u\|_{\dot{B}_{p, r}^{s}}<\infty\right\}$.

- If $k \in \mathbb{N}$ and $\frac{3}{p}+k \leq s<\frac{3}{p}+k+1$ (or $s=\frac{3}{p}+k+1$ if $r=1$ ), then $\dot{B}_{p, r}^{s}\left(\mathbb{R}^{3}\right)$ is defined as the subset of distributions $u \in \mathcal{S}^{\prime}\left(\mathbb{R}^{3}\right)$ such that $\partial^{\beta} u \in \dot{B}_{p, r}^{s-k}\left(\mathbb{R}^{3}\right)$ whenever $|\beta|=k$.

Lemma 2.1 (Bernstein-type lemma [11-13]) Let $\mathcal{B}$ be a ball and $\mathcal{C}$ a ring of $\mathbb{R}^{3}$. A constant $C$ exists so that for any positive real number $\lambda$, any non-negative integer $k$, any smooth homogeneous function $\sigma$ of degree $m$, and any couple of real numbers $(a, b)$ with $b \geq a \geq 1$, we have

$$
\begin{aligned}
& \operatorname{Supp} \hat{u} \subset \lambda \mathcal{B} \Rightarrow \sup _{|\alpha|=k}\left\|\partial^{\alpha} u\right\|_{L^{b}} \leq C^{k+1} \lambda^{k+3\left(\frac{1}{a}-\frac{1}{b}\right)}\|u\|_{L^{a}}, \\
& \text { Supp } \hat{u} \subset \lambda \mathcal{C} \Rightarrow C^{-1-k} \lambda^{k}\|u\|_{L^{a}} \leq \sup _{|\alpha|=k}\left\|\partial^{\alpha} u\right\|_{L^{a}} \leq C^{1+k} \lambda^{k}\|u\|_{L^{a}} .
\end{aligned}
$$

Definition 2.2 (see $[14,15]$ ) Let $s \leq \frac{3}{p}$ (respectively, $s \in \mathbb{R}$ ), $(r, \lambda, p) \in[1,+\infty]^{3}$, and $T \in$ ] $0,+\infty]$. We define $\widetilde{L}_{T}^{\lambda}\left(\dot{B}_{p r}^{s}\left(\mathbb{R}^{3}\right)\right)$ as the completion of $C\left([0, T], \mathcal{S}\left(\mathbb{R}^{3}\right)\right)$ by the norm

$$
\|f\|_{\tilde{L}_{T}^{\lambda}\left(\dot{B}_{p, r}^{s}\right)} \stackrel{\text { def }}{=}\left(\sum_{q \in \mathbb{Z}} 2^{q r s}\left(\int_{0}^{T}\left\|\dot{\Delta}_{q} f(t)\right\|_{L^{p}}^{\lambda} d t\right)^{\frac{r}{\lambda}}\right)^{\frac{1}{r}}<\infty,
$$

with the usual change if $r=\infty$. For brevity, we just denote this space by $\widetilde{L}_{T}^{\lambda}\left(\dot{B}_{p, r}^{s}\right)$. In the particular case when $p=r=2$, we denote this space by $\widetilde{L}_{T}^{\lambda}\left(\dot{H}^{s}\right)$.

\section{Global existence of (1.2)}

We start with the local existence of strong solutions whose proof can be found in Theorem 1.1 of [16].

Lemma 3.1 Under the assumptions of Theorem 1.1, a positive time $T$ exists so that (1.2) has a unique local solution $a \in \mathcal{C}_{b}\left([0, T] ; B_{2,1}^{3 / 2}\left(\mathbb{R}^{3}\right)\right) ; u, B \in \mathcal{C}_{b}\left([0, T] ; \dot{B}_{2,1}^{1 / 2}\left(\mathbb{R}^{3}\right)\right) \cap$ $L^{1}\left([0, T] ; \dot{B}_{2,1}^{5 / 2}\left(\mathbb{R}^{3}\right)\right)$ and, for $T \geq 1$, we have

$$
\|(u, B)\|_{\widetilde{L}^{\infty}\left([0, T] ; \dot{B}_{2,1}^{1 / 2}\right)}+\int_{0}^{T}\|(u, B)(\tau)\|_{\dot{B}_{2,1}^{5 / 2}} \delta \tau \leq C\left(\left\|u_{0}\right\|_{\dot{B}_{2,1}^{1 / 2}}+\left\|B_{0}\right\|_{\dot{B}_{2,1}^{1 / 2}}\right) .
$$

Similar to Theorem 2 in [8], we can obtain a higher-order regularity of the local solution to (1.2) as follows. 
Proposition 3.1 Under the assumptions of Theorem 1.1, for any $t_{0}>0$, we have

$$
\begin{aligned}
& \|(u, B)\|_{\widetilde{L}^{\infty}\left(\left[t_{0}, T\right] ; \dot{B}_{2,1}^{3 / 2}\right)}+\|(u, B)\|_{L^{1}\left(\left[t_{0}, T\right] ; \dot{B}_{2,1}^{7 / 2}\right)}+\|\nabla \Pi\|_{L^{1}\left(\left[t_{0}, T\right] ; \dot{B}_{2,1}^{3 / 2}\right)} \\
& \quad \leq C\left(\left\|a_{0}\right\|_{B_{2,1}^{3 / 2}}\right)\left(\left\|u_{0}\right\|_{\dot{B}_{2,1}^{1 / 2}}+\left\|B_{0}\right\|_{\dot{B}_{2,1}^{1 / 2}}\right)\left(1+1 / \sqrt{t_{0}}\right) \exp \left\{C\left(\left\|u_{0}\right\|_{\dot{B}_{2,1}^{1 / 2}}+\left\|B_{0}\right\|_{\dot{B}_{2,1}^{1 / 2}}\right)\right\} .
\end{aligned}
$$

Remark 3.1 Thanks to (3.1) and (3.2), there exists $t_{1} \in(0,1)$ so that $u\left(t_{1}\right), B\left(t_{1}\right) \in \dot{B}_{2,1}^{1 / 2}\left(\mathbb{R}^{3}\right) \cap$ $\dot{B}_{2,1}^{7 / 2}\left(\mathbb{R}^{3}\right)$ and it satisfies (1.6).

We are in a position to prove Theorem 1.1.

Proof of Theorem 1.1 Thanks to Lemma 3.1, we conclude that: given $a_{0} \in B_{2,1}^{3 / 2}\left(\mathbb{R}^{3}\right), u_{0}, B_{0} \in$ $\dot{B}_{2,1}^{1 / 2}\left(\mathbb{R}^{3}\right)$ with $\left\|u_{0}\right\|_{\dot{B}_{2,1}^{1 / 2}}+\left\|B_{0}\right\|_{\dot{B}_{2,1}^{1 / 2}}$ sufficiently small, (1.2) has a unique local solution $(a, u)$ satisfying $a \in \mathcal{C}\left(\left[0, T^{*}\right) ; B_{2,1}^{3 / 2}\left(\mathbb{R}^{3}\right)\right)$, and $u, B \in \mathcal{C}\left(\left[0, T^{*}\right) ; \dot{B}_{2,1}^{1 / 2}\left(\mathbb{R}^{3}\right)\right) \cap L_{\text {loc }}^{1}\left(\left(0, T^{*}\right) ; \dot{B}_{2,1}^{5 / 2}\left(\mathbb{R}^{3}\right)\right)$ for some $T^{*}>1$. Our aim o is to prove that $T^{*}=\infty$.

As $\left\|u\left(t_{1}\right)\right\|_{\dot{B}_{2,1}^{1 / 2} \cap \dot{B}_{2,1}^{7 / 2}},\left\|B\left(t_{1}\right)\right\|_{\dot{B}_{2,1}^{1 / 2} \cap \dot{B}_{2,1}^{7 / 2}}$ is very small provided $\left\|u_{0}\right\|_{\dot{B}_{2,1}^{1 / 2}},\left\|B_{0}\right\|_{\dot{B}_{2,1}^{1 / 2}}$ is sufficiently small. Let $v$ solve the classical Navier-Stokes system (1.7). As $u\left(t_{1}\right)$ is sufficient small in $\dot{B}_{2,1}^{1 / 2}\left(\mathbb{R}^{3}\right)$, it follows from the classical theory of Navier-Stokes equations [15] that (1.7) has a unique global solution $v \in \mathcal{C}\left(\left[t_{1},+\infty\right) ; \dot{B}_{2,1}^{1 / 2}\right) \cap L^{1}\left(\left[t_{1},+\infty\right) ; \dot{B}_{2,1}^{5 / 2}\right)$ satisfying

$$
\begin{aligned}
& \|v\|_{\widetilde{L}^{\infty}\left(\left[t_{1},+\infty\right) ; \dot{B}_{2,1}^{1 / 2}\right)}+\|v\|_{L^{1}\left(\left[t_{1},+\infty\right) ; \dot{B}_{2,1}^{5 / 2}\right)}+\left\|\nabla \Pi_{v}\right\|_{L^{1}\left(\left[t_{1},+\infty\right) ; \dot{B}_{2,1}^{1 / 2}\right)} \leq C\left\|u\left(t_{1}\right)\right\|_{\dot{B}_{2,1}^{1 / 2}}, \\
& \left\|\partial_{t} v\right\|_{L^{1}\left(\left[t_{1},+\infty\right) ; \dot{B}_{2,1}^{1 / 2}\right)} \leq C\left\|u\left(t_{1}\right)\right\|_{\dot{B}_{2,1}^{1 / 2}}+\|\operatorname{div}(v \otimes v)\|_{L^{1}\left(\left[t_{1},+\infty\right) ; \dot{B}_{2,1}^{1 / 2}\right)} \leq C\left\|u\left(t_{1}\right)\right\|_{\dot{B}_{2,1}^{1 / 2}}
\end{aligned}
$$

With $v$ thus obtained, we denote $w \stackrel{\text { def }}{=} u-v$. Then, thanks to (1.3) and (1.7), $w, B$ solves (1.8). Then the proof of Theorem 1.1 reduces to proving the global well-posedness of (1.8). For simplicity, we just present the a priori estimates for smooth enough solutions of (1.8) on $\left[0, T^{*}\right)$.

\subsection{The higher regularities of $v$}

Proposition $3.2([8])$ Let $\left(v, \Pi_{v}\right)$ be the unique global solution of (1.7) which satisfies (3.3). Then, for $s_{1} \in\left[\frac{3}{2}, \frac{7}{2}\right]$ and $s_{2} \in\left[\frac{1}{2}, \frac{3}{2}\right]$, we have

$$
\begin{aligned}
& \|v\|_{\widetilde{L}^{\infty}\left(\left[t_{1},+\infty\right) ; \dot{B}_{2,1}^{\left.s_{1}\right)}\right.}+\left\|\left(\Delta v, \nabla \Pi_{v}\right)\right\|_{L^{1}\left(\left[t_{1},+\infty\right) ; \dot{B}_{2,1}^{s_{1}}\right)} \leq C\left\|u_{0}\right\|_{\dot{B}_{2,1}^{1 / 2}}, \\
& \left\|\partial_{t} v\right\|_{\widetilde{L}^{\infty}\left(\left[t_{1},+\infty\right) ; \dot{B}_{2,1}^{s_{2}}\right)}+\left\|\left(\partial_{t} \Delta v, \partial_{t} \nabla \Pi_{v}\right)\right\|_{L^{1}\left(\left[t_{1},+\infty\right) ; \dot{B}_{2,1}^{s_{2}}\right)} \leq C\left\|u_{0}\right\|_{\dot{B}_{2,1}^{1 / 2}} .
\end{aligned}
$$

Corollary 3.1 ([8]) Under the assumptions of Proposition 3.2, one has

$$
\|\nabla v\|_{L^{2}\left(\left[t_{1},+\infty\right) ; L^{\infty}\right)}+\left\|\Delta v-\nabla \Pi_{v}\right\|_{L^{2}\left(\left[t_{1},+\infty\right) ; L^{\infty}\right)} \leq C\left\|u_{0}\right\|_{\dot{B}_{2,1}^{1 / 2}}
$$

\subsection{The estimate of $(w, B)$}

Lemma 3.2 $\left(L^{2}\right.$ estimate of $\left.(w, B)\right)$ We have for $t_{1}<t<T^{*}$,

$$
\begin{aligned}
& \|w\|_{L^{\infty}\left(\left[t_{1}, t\right] ; L^{2}\right)}+\|\nabla w\|_{L^{2}\left(\left[t_{1}, t\right] ; L^{2}\right)}+\|B\|_{L^{\infty}\left(\left[t_{1}, t\right] ; L^{2}\right)}+\|\nabla B\|_{L^{2}\left(\left[t_{1}, t\right] ; L^{2}\right)} \\
& \quad \leq C\left(\left\|u_{0}\right\|_{\dot{B}_{2,1}^{1 / 2}}+\left\|B_{0}\right\|_{\left.\dot{B}_{2,1}^{1 / 2}\right)}\right.
\end{aligned}
$$

with $C$ being independent of $t$. 
Proof First of all, thanks to (1.4), one deduces from the transport equation of (1.8) that

$$
\left(1+\left\|a_{0}\right\|_{\dot{B}_{2,1}^{3 / 2}}\right)^{-1} \leq \rho(t, x) \leq \underline{b}^{-1},
$$

from which, with $1-\rho=\rho a$, we get by using the standard energy estimate to the $w, B$ equation of (1.8)

$$
\begin{aligned}
& \frac{1}{2} \frac{d}{d t}\|\sqrt{\rho} w\|_{L^{2}}^{2}+\|\nabla w\|_{L^{2}}^{2}=\int_{\mathbb{R}^{3}}\left((1-\rho)\left(\partial_{t} v+v \cdot \nabla v\right)+\rho w \cdot \nabla v+B \cdot \nabla B\right) \cdot w d x, \\
& \frac{1}{2} \frac{d}{d t}\|B\|_{L^{2}}^{2}+\|\nabla B\|_{L^{2}}^{2}=\int_{\mathbb{R}^{3}}(B \cdot \nabla v \cdot B+B \cdot \nabla w \cdot B) d x ;
\end{aligned}
$$

thanks to $\operatorname{div} B=0$, one has

$$
\int_{\mathbb{R}^{3}} B \cdot \nabla w \cdot B d x+\int_{\mathbb{R}^{3}} B \cdot \nabla B \cdot w d x=0 .
$$

Therefore, we get

$$
\begin{aligned}
& \frac{1}{2} \frac{d}{d t}\left(\|\sqrt{\rho} w\|_{L^{2}}^{2}+\|B\|_{L^{2}}^{2}\right)+\|\nabla w\|_{L^{2}}^{2}+\|\nabla B\|_{L^{2}}^{2} \\
& \quad=\int_{\mathbb{R}^{3}}\left((1-\rho)\left(\partial_{t} v+v \cdot \nabla v\right) \cdot w+\rho w \cdot \nabla v \cdot w+B \cdot \nabla v \cdot B\right) d x \\
& \quad \leq C\left(\|\sqrt{\rho} w\|_{L^{2}}\|a\|_{L^{2}}\left\|\partial_{t} v+v \cdot \nabla v\right\|_{L^{\infty}}+\|\nabla v\|_{L^{\infty}}\left(\|\sqrt{\rho} w\|_{L^{2}}^{2}+\|B\|_{L^{2}}^{2}\right)\right),
\end{aligned}
$$

from which we get

$$
\begin{aligned}
& \frac{1}{2} \frac{d}{d t}\left(\|\sqrt{\rho} w\|_{L^{2}}^{2}+\|B\|_{L^{2}}^{2}\right) \\
& \quad \leq C\left(\|\sqrt{\rho} w\|_{L^{2}}\|a\|_{L^{2}}\left\|\partial_{t} v+v \cdot \nabla v\right\|_{L^{\infty}}+\|\nabla v\|_{L^{\infty}}\left(\|\sqrt{\rho} w\|_{L^{2}}^{2}+\|B\|_{L^{2}}^{2}\right)\right) \\
& \quad \leq C\left(\left(\|\sqrt{\rho} w\|_{L^{2}}+\|B\|_{L^{2}}\right)\|a\|_{L^{2}}\left\|\partial_{t} v+v \cdot \nabla v\right\|_{L^{\infty}}+\|\nabla v\|_{L^{\infty}}\left(\|\sqrt{\rho} w\|_{L^{2}}^{2}+\|B\|_{L^{2}}^{2}\right)\right)
\end{aligned}
$$

from which we infer for $t \in\left(t_{1}, T^{*}\right)$ that

$$
\begin{aligned}
& \frac{d}{d t}\left(e^{-2 \int_{t_{1}}^{t}\|\nabla v(\tau)\|_{L^{\infty}} d \tau}\left(\|\sqrt{\rho} w(t)\|_{L^{2}}^{2}+\|B(t)\|_{L^{2}}^{2}\right)\right) \\
& \quad \leq C\left\|a_{0}\right\|_{L^{2}} e^{-2 \int_{t_{1}}^{t}\|\nabla v(\tau)\|_{L^{\infty}} d \tau}\left(\|\sqrt{\rho} w\|_{L^{2}}+\|B(t)\|_{L^{2}}\right)\left\|\Delta v-\nabla \Pi_{v}\right\|_{\dot{B}_{2,1}^{3 / 2}}
\end{aligned}
$$

This along with (1.6) and (3.4) implies

$$
\begin{aligned}
\|\sqrt{\rho} w\|_{L^{\infty}\left(\left[t_{1}, t\right] ; L^{2}\right)}^{2}+\|B(t)\|_{L^{\infty}\left(\left[t_{1}, t\right] L^{2}\right)}^{2} & \leq C e^{\int_{t_{1}}^{t}\|\nabla v(\tau)\|_{L^{\infty}} d \tau}\left\|\Delta v-\nabla \Pi_{v}\right\|_{L^{1}\left(\left[t_{1}, t\right], \dot{B}_{2,1}^{3 / 2}\right)} \\
& \leq C\left\|u\left(t_{1}\right)\right\|_{\dot{B}_{2,1}^{3 / 2}} \exp \left\{C\left\|u\left(t_{0}\right)\right\|_{\dot{B}_{2,1}^{1 / 2}}\right\} \\
& \leq C\left(\left\|u_{0}\right\|_{\dot{B}_{2,1}^{1 / 2}}+\left\|B_{0}\right\|_{\dot{B}_{2,1}^{1 / 2}}\right) .
\end{aligned}
$$

Plugging the above estimate into (3.9) gives rise to

$$
\|\nabla w\|_{L^{2}\left(\left[t_{1}, t\right] ; L^{2}\right)}+\|\nabla B\|_{L^{2}\left(\left[t_{1}, t\right] ; L^{2}\right)} \leq C\left(\left\|u_{0}\right\|_{\dot{B}_{2,1}^{1 / 2}}+\left\|B_{0}\right\|_{\dot{B}_{2,1}^{1 / 2}}\right) .
$$

This completes the proof of Lemma 3.2. 
Lemma $3.3\left(H^{1}\right.$ estimate of $\left.(w, B)\right)$ There exist two positive constants $c_{1}$ and $c_{2}$ such that for $t \in\left[t_{1}, T^{*}\right)$,

$$
\begin{aligned}
& \|(\nabla w, \nabla B)\|_{L^{\infty}\left(\left[t_{1}, t\right], L^{2}\right)}^{2}+\int_{t_{1}}^{t}\left(c_{1}\left\|\partial_{t} w\right\|_{L^{2}}^{2}+c_{2}\left\|\left(\nabla^{2} B, \nabla^{2} w\right)\right\|_{L^{2}}^{2}+\left\|\nabla \Pi_{w}\right\|_{L^{2}}^{2}\right) d t^{\prime} \\
& \quad \leq C\left\|u_{0}\right\|_{\dot{B}_{2,1}^{1 / 2}}^{2}
\end{aligned}
$$

with $C$ being independent of $t$.

Proof Taking the $L^{2}$ inner product of the $w$ equation of (1.8) with $\frac{1}{\rho} \Delta w$ and using (3.8), we obtain

$$
\begin{aligned}
\frac{1}{2} \frac{d}{d t}\|\nabla w\|_{L^{2}}^{2}+\left\|\frac{1}{\sqrt{\rho}} \Delta w\right\|_{L^{2}}^{2} \leq & C\left\|\frac{1}{\sqrt{\rho}} \Delta w\right\|_{L^{2}}\left\{\|\nabla w\|_{L^{2}}\|v\|_{L^{\infty}}+\|\nabla w\|_{L^{6}}\|w\|_{L^{3}}\right. \\
& +\|w\|_{L^{2}}\|\nabla v\|_{L^{\infty}}+\left\|\nabla \Pi_{w}\right\|_{L^{2}} \\
& \left.+\|a\|_{L^{2}}\left\|\partial_{t} v+v \cdot \nabla v\right\|_{L^{\infty}}+\|\nabla B\|_{L^{6}}\|B\|_{L^{3}}\right\},
\end{aligned}
$$

which implies

$$
\begin{aligned}
\frac{d}{d t}\|\nabla w\|_{L^{2}}^{2}+\left\|\frac{1}{\sqrt{\rho}} \Delta w\right\|_{L^{2}}^{2} \leq & C\|\nabla w\|_{L^{2}}^{2}\|v\|_{L^{\infty}}^{2}+\|\Delta w\|_{L^{2}}^{2}\|w\|_{L^{3}}^{2}+\|w\|_{L^{2}}^{2}\|\nabla v\|_{L^{\infty}}^{2} \\
& +\left\|\nabla \Pi_{w}\right\|_{L^{2}}^{2}+\left\|\Delta v-\nabla \Pi_{v}\right\|_{L^{\infty}}^{2}+\|\Delta B\|_{L^{2}}^{2}\|B\|_{L^{3}}^{2} .
\end{aligned}
$$

Again thanks to the $w$ equation of (1.8) and $\operatorname{div} w=0$, one has

$$
\begin{aligned}
\|\Delta w\|_{L^{2}}^{2}+\left\|\nabla \Pi_{w}\right\|_{L^{2}}^{2}= & \left\|\Delta w-\nabla \Pi_{w}\right\|_{L^{2}}^{2} \\
\leq & C\left\{\left\|\sqrt{\rho} \partial_{t} w(t)\right\|_{L^{2}}^{2}+\|w(t)\|_{L^{3}}^{2}\|\Delta w(t)\|_{L^{2}}^{2}+\|v\|_{L^{\infty}}^{2}\|\nabla w\|_{L^{2}}^{2}\right. \\
& \left.+\left\|\Delta v-\nabla \Pi_{v}\right\|_{L^{\infty}}^{2}+\|w\|_{L^{2}}^{2}\|\nabla v\|_{L^{\infty}}^{2}+\|B(t)\|_{L^{3}}^{2}\|\Delta B(t)\|_{L^{2}}^{2}\right\} .
\end{aligned}
$$

As a consequence, we obtain for some positive constant $c_{1}$,

$$
\begin{aligned}
\frac{d}{d t}\|\nabla w\|_{L^{2}}^{2}+c_{1}\left\|\nabla^{2} w\right\|_{L^{2}}^{2} \leq & C\left\{\|w\|_{L^{3}}^{2}\left\|\nabla^{2} w\right\|_{L^{2}}^{2}+\left\|\sqrt{\rho} \partial_{t} w\right\|_{L^{2}}^{2}\right. \\
& +\|\nabla w\|_{L^{2}}^{2}\|v\|_{L^{\infty}}^{2}+\|w\|_{L^{2}}^{2}\|\nabla v\|_{L^{\infty}}^{2} \\
& \left.+\left\|\Delta v-\nabla \Pi_{v}\right\|_{L^{\infty}}^{2}+\|\Delta B\|_{L^{2}}^{2}\|B\|_{L^{3}}^{2}\right\}
\end{aligned}
$$

Taking the $L^{2}$ inner product of the $B$ equation of (1.8) with $\Delta B$,

$$
\begin{aligned}
\frac{d}{d t}\|\nabla B\|_{L^{2}}^{2}+\left\|\nabla^{2} B\right\|_{L^{2}}^{2} \leq & C\left\{\|B\|_{L^{3}}^{2}\left\|\nabla^{2} w\right\|_{L^{2}}^{2}+\|w\|_{L^{3}}^{2}\left\|\nabla^{2} B\right\|_{L^{2}}^{2}\right. \\
& \left.+\|\nabla B\|_{L^{2}}^{2}\|v\|_{L^{\infty}}^{2}+\|B\|_{L^{2}}^{2}\|\nabla v\|_{L^{\infty}}^{2}\right\} .
\end{aligned}
$$

Along the same lines, we get by taking the $L^{2}$ inner product of the $w$ equation of (1.8) with $\partial_{t} w$,

$$
\begin{aligned}
\frac{d}{d t}\|\nabla w\|_{L^{2}}^{2}+\left\|\sqrt{\rho} \partial_{t} w\right\|_{L^{2}}^{2} \leq & C\left(\|w\|_{L^{3}}^{2}\left\|\nabla^{2} w\right\|_{L^{2}}^{2}+\|\nabla w\|_{L^{2}}^{2}\|v\|_{L^{\infty}}^{2}+\|w\|_{L^{2}}^{2}\|\nabla v\|_{L^{\infty}}^{2}\right. \\
& \left.+\left\|\Delta v-\nabla \Pi_{v}\right\|_{L^{\infty}}^{2}+\|\Delta B\|_{L^{2}}^{2}\|B\|_{L^{3}}^{2}\right) .
\end{aligned}
$$


Thanks to (3.11), (3.12), and (3.13), there is a positive constant $c_{2}$ so that

$$
\begin{aligned}
\frac{d}{d t}\left(\|\nabla w\|_{L^{2}}^{2}+\|\nabla B\|_{L^{2}}^{2}\right)+c_{2}\left\|\partial_{t} w\right\|_{L^{2}}^{2} \\
\quad+\left(C_{3}-C_{2}\left(\|w\|_{L^{3}}^{2}+\|B\|_{L^{3}}^{2}\right)\right)\left(\left\|\nabla^{2} w\right\|_{L^{2}}^{2}+\left\|\nabla^{2} B\right\|_{L^{2}}^{2}\right) \\
\leq C_{4}\left(\|\nabla w\|_{L^{2}}^{2}\|v\|_{L^{\infty}}^{2}+\|w\|_{L^{2}}^{2}\|\nabla v\|_{L^{\infty}}^{2}+\left\|\Delta v-\nabla \Pi_{v}\right\|_{L^{\infty}}^{2}\right. \\
\left.\quad+\|\nabla B\|_{L^{2}}^{2}\|v\|_{L^{\infty}}^{2}+\|B\|_{L^{2}}^{2}\|\nabla v\|_{L^{\infty}}^{2}\right) \\
\leq C_{4}\left(\left(\|\nabla w\|_{L^{2}}^{2}+\|\nabla B\|_{L^{2}}^{2}\right)\|v\|_{L^{\infty}}^{2}\right. \\
\left.\quad+\left(\|w\|_{L^{2}}^{2}+\|B\|_{L^{2}}^{2}\right)\|\nabla v\|_{L^{\infty}}^{2}+\left\|\Delta v-\nabla \Pi_{v}\right\|_{L^{\infty}}^{2}\right) .
\end{aligned}
$$

Now let $\tau^{*}$ be determined by

$$
\tau^{*} \stackrel{\text { def }}{=} \sup \left\{t \geq t_{1},\|w(t)\|_{L^{3}}^{2}+\|B(t)\|_{L^{3}}^{2} \leq \frac{C_{3}}{2 C_{2}}\right\} .
$$

We claim that $\tau^{*}=T^{*}$ provided that $\left\|u_{0}\right\|_{\dot{B}_{2,1}^{1 / 2}}+\left\|B_{0}\right\|_{\dot{B}_{2,1}^{1 / 2}}$ is sufficiently small. Otherwise for $t \in\left[t_{1}, \tau^{*}\right)$, it follows from (3.14) that

$$
\begin{aligned}
& \frac{d}{d t}\left(\|\nabla w\|_{L^{2}}^{2}+\|\nabla B\|_{L^{2}}^{2}\right)+c_{2}\left\|\partial_{t} w\right\|_{L^{2}}^{2}+\frac{C_{3}}{2}\left(\left\|\nabla^{2} w\right\|_{L^{2}}^{2}+\left\|\nabla^{2} B\right\|_{L^{2}}^{2}\right) \\
& \quad \leq C_{4}\left(\left(\|\nabla w\|_{L^{2}}^{2}+\|\nabla B\|_{L^{2}}^{2}\right)\|v\|_{L^{\infty}}^{2}\right. \\
& \left.\quad+\left(\|w\|_{L^{2}}^{2}+\|B\|_{L^{2}}^{2}\right)\|\nabla v\|_{L^{\infty}}^{2}+\left\|\Delta v-\nabla \Pi_{v}\right\|_{L^{\infty}}^{2}\right) .
\end{aligned}
$$

Applying Gronwall's inequality to (3.16) and using (3.7), (3.6), (1.6), and (3.3) together with the interpolation inequality yield

$$
\begin{aligned}
& \|\nabla w\|_{L^{2}}^{2}+\|\nabla B\|_{L^{2}}^{2} \\
& \quad \leq C_{4} \exp \left\{C_{4} \int_{t_{1}}^{t}\|v\|_{L^{\infty}}^{2} d t^{\prime}\right\}\left[\left\|\nabla B\left(t_{1}\right)\right\|_{L^{2}}^{2}+\int_{t_{1}}^{t}\left(\|\nabla v\|_{L^{\infty}}^{2}+\left\|\Delta v-\nabla \Pi_{v}\right\|_{L^{\infty}}^{2}\right) d t^{\prime}\right] \\
& \quad \leq C_{5}\left(\left\|u_{0}\right\|_{\dot{B}_{2,1}^{1 / 2}}^{2}+\left\|B_{0}\right\|_{\dot{B}_{2,1}^{1 / 2}}^{2}\right) .
\end{aligned}
$$

However, notice from (3.7) and (3.17) that

$$
\begin{aligned}
\|w(t)\|_{L^{3}}^{2}+\|B(t)\|_{L^{3}}^{2} & \leq C\left(\|w(t)\|_{L^{2}}\|\nabla w(t)\|_{L^{2}}+\|B(t)\|_{L^{2}}\|\nabla B(t)\|_{L^{2}}\right) \\
& \leq C_{6}\left(\left\|u_{0}\right\|_{\dot{B}_{2,1}^{1 / 2}}^{2}+\left\|B_{0}\right\|_{\dot{B}_{2,1}^{1 / 1}}^{2}\right) \leq \frac{C_{3}}{4 C_{2}} \quad \text { for } t \in\left[t_{1}, \tau^{*}\right)
\end{aligned}
$$

provided that $\left\|u_{0}\right\|_{\dot{B}_{2,1}^{1 / 2}}^{2}+\left\|B_{0}\right\|_{\dot{B}_{2,1}^{1 / 2}}^{2} \leq \frac{C_{3}}{4 C_{2} C_{6}}$, which contradicts with (3.15). This in turn shows that $\tau^{*}=T^{*}$. Then integrating (3.16) and using (3.6) leads to (3.10). This completes the proof of the lemma.

Lemma 3.4 $\left(H^{2}\right.$ estimate of $\left.(w, B)\right)$ There exists a time independent constant $C$ so that for $t \in\left[t_{1}, T^{*}\right)$,

$$
\left\|\left(\nabla^{2} w, \nabla^{2} B\right)\right\|_{L^{\infty}\left(\left[t_{1}, t\right], L^{2}\right)}+\left\|\left(\nabla w_{t}, \nabla B_{t}\right)\right\|_{L^{2}\left(\left[t_{1}, t\right], L^{2}\right)}+\left\|\left(\nabla^{2} w, \nabla^{2} B\right)\right\|_{L^{2}\left(\left[t_{1}, t\right], L^{6}\right)} \leq C .
$$


Proof Step 1. $L^{2}$ estimate of $\left(\sqrt{\rho} w_{t}, B_{t}\right)$.

We get by first acting $\partial_{t}$ to the $w$ equation of (1.8) and then taking the $L^{2}$ inner product of the resulting equation with $\partial_{t} w$,

$$
\begin{aligned}
\frac{1}{2} \frac{d}{d t}\left\|\sqrt{\rho} w_{t}\right\|_{L^{2}}^{2}+\left\|\nabla w_{t}\right\|_{L^{2}}^{2} & \int_{\mathbb{R}^{3}}(1-\rho) w_{t} \cdot \partial_{t}\left(\Delta v-\nabla \Pi_{v}\right) d x \\
& \quad-\int_{\mathbb{R}^{3}} \rho_{t} w_{t} \cdot\left(w_{t}+(w+v) \cdot \nabla w+w \cdot \nabla v+\left(\Delta v-\nabla \Pi_{v}\right)\right) d x \\
& \quad-\int_{\mathbb{R}^{3}} \rho w_{t} \cdot\left((v+w)_{t} \cdot \nabla w+w_{t} \cdot \nabla v+w \cdot \nabla v_{t}\right) d x \\
& +\int_{\mathbb{R}^{3}} \partial_{t}(B \cdot \nabla B) w_{t} d x \\
\stackrel{\operatorname{def}}{=} & I+I I+I I I+I V .
\end{aligned}
$$

The estimates of $I, I I$, and $I I I$ are similar to [8],

$$
\begin{aligned}
|I| \leq & C\left\|a_{0}\right\|_{\dot{B}_{2,1}^{3 / 2}}\left\|\sqrt{\rho} w_{t}\right\|_{L^{2}}\left\|\partial_{t}\left(\Delta v-\nabla \Pi_{v}\right)\right\|_{L^{2}}, \\
|I I| \leq & \frac{1}{4}\left\|\nabla w_{t}\right\|_{L^{2}}^{2}+C\left\{\|v\|_{L^{\infty}}^{4}+\|\nabla v\|_{L^{6}}^{2}+\|\Delta w\|_{L^{2}}^{2}+\left\|\Delta v-\nabla \Pi_{v}\right\|_{L^{4}}^{2}\right. \\
& +\left\|\nabla^{2} v\right\|_{L^{6}}^{2}+\left\|\sqrt{\rho} w_{t}\right\|_{L^{2}}^{2}\left(\|v\|_{L^{\infty}}^{2}+\|\nabla w\|_{L^{2}}\left\|\nabla^{2} w\right\|_{L^{2}}\right) \\
& \left.+\left\|\sqrt{\rho} w_{t}\right\|_{L^{2}}\left\|\nabla\left(\Delta v-\nabla \Pi_{v}\right)\right\|_{L^{4}}\right\}, \\
|I I I| \leq & \frac{1}{16}\left\|\nabla w_{t}\right\|_{L^{2}}^{2}+C\left\{\left\|\sqrt{\rho} w_{t}\right\|_{L^{2}}^{2}\left(\|\nabla w\|_{L^{2}}\left\|\nabla^{2} w\right\|_{L^{2}}+\|\nabla v\|_{L^{\infty}}\right)\right. \\
& \left.+\left\|\sqrt{\rho} w_{t}\right\|_{L^{2}}\left(\left\|v_{t}\right\|_{L^{\infty}}+\left\|\nabla v_{t}\right\|_{L^{4}}\right)\right\},
\end{aligned}
$$

which, thanks to (3.10), yields

$$
\begin{aligned}
|I V| & =\left|\int_{\mathbb{R}^{3}} B_{t} \cdot \nabla B \cdot w_{t} d x+\int_{\mathbb{R}^{3}} B \cdot \nabla B_{t} \cdot w_{t} d x\right| \\
& \leq \varepsilon\left\|\nabla w_{t}\right\|_{L^{2}}^{2}+C_{\varepsilon}\left\|B_{t}\right\|_{L^{2}}^{2}\|\nabla B\|_{L^{2}}\left\|\nabla^{2} B\right\|_{L^{2}} .
\end{aligned}
$$

This together with (3.19), (3.20), (3.21), and (3.22) yields

$$
\begin{aligned}
\frac{d}{d t} \| & \sqrt{\rho} w_{t}\left\|_{L^{2}}^{2}+\right\| \nabla w_{t} \|_{L^{2}}^{2} \\
\leq & C\left\|\sqrt{\rho} w_{t}\right\|_{L^{2}}\left[\left\|\nabla\left(\Delta v-\nabla \Pi_{v}\right)\right\|_{L^{4}}\right. \\
& \left.+\left\|\partial_{t}\left(\Delta v-\nabla \Pi_{v}\right)\right\|_{L^{2}}+\left\|v_{t}\right\|_{L^{\infty}}+\left\|\nabla v_{t}\right\|_{L^{4}}\right] \\
& +C\left\|\sqrt{\rho} w_{t}\right\|_{L^{2}}^{2}\left[\|v\|_{L^{\infty}}^{2}+\|\nabla v\|_{L^{\infty}}+\|\nabla w\|_{L^{2}}\left\|\nabla^{2} w\right\|_{L^{2}}\right] \\
& +C\left[\|v\|_{L^{\infty}}^{4}+\|\nabla v\|_{L^{6}}^{2}+\|\Delta w\|_{L^{2}}^{2}+\left\|\Delta v-\nabla \Pi_{v}\right\|_{L^{4}}^{2}+\left\|\nabla^{2} v\right\|_{L^{6}}^{2}\right] \\
& +C_{\varepsilon}\left\|B_{t}\right\|_{L^{2}}^{2}\left\|\nabla^{2} B\right\|_{L^{2}}\|\nabla B\|_{L^{2}} .
\end{aligned}
$$


On the other hand, acting by $\partial_{t}$ to the $B$ equation of (1.8) and then taking the $L^{2}$ inner product of the resulting equation with $\partial_{t} B$ we see that

$$
\begin{aligned}
& \frac{1}{2} \frac{d}{d t}\left\|B_{t}\right\|_{L^{2}}^{2}+\left\|\nabla B_{t}\right\|_{L^{2}}^{2} \\
& =\int_{\mathbb{R}^{3}} B_{t} \cdot \nabla(v+w) \cdot B_{t}+B \cdot \nabla(v+w)_{t} \cdot B_{t}-(v+w)_{t} \cdot \nabla B \cdot B_{t} d x \\
& \leq \varepsilon\left(\left\|\nabla w_{t}\right\|_{L^{2}}^{2}+\left\|\nabla B_{t}\right\|_{L^{2}}^{2}\right)+C\left\|B_{t}\right\|_{L^{2}}^{2}\left(\|\nabla w\|_{L^{2}}\left\|\nabla^{2} w\right\|_{L^{2}}\right. \\
& \left.\quad+\|\nabla B\|_{L^{2}}\left\|\nabla^{2} B\right\|_{L^{2}}+\|\nabla v\|_{L^{\infty}}\right)+C\left\|B_{t}\right\|_{L^{2}}\left(\left\|\nabla v_{t}\right\|_{L^{4}}+\left\|v_{t}\right\|_{L^{\infty}}\right) .
\end{aligned}
$$

Thanks to (3.23) and (3.24), we have

$$
\begin{aligned}
\frac{1}{2} \frac{d}{d t}( & \left.\left\|\sqrt{\rho} w_{t}\right\|_{L^{2}}^{2}+\left\|B_{t}\right\|_{L^{2}}^{2}\right)+\left\|\nabla B_{t}\right\|_{L^{2}}^{2}+\left\|\nabla w_{t}\right\|_{L^{2}}^{2} \\
\leq & C\left(\left\|\sqrt{\rho} w_{t}\right\|_{L^{2}}+\left\|B_{t}\right\|_{L^{2}}\right) \\
& \times\left[\left\|\nabla\left(\Delta v-\nabla \Pi_{v}\right)\right\|_{L^{4}}+\left\|\partial_{t}\left(\Delta v-\nabla \Pi_{v}\right)\right\|_{L^{4}}+\left\|v_{t}\right\|_{L^{\infty}}+\left\|\nabla v_{t}\right\|_{L^{4}}\right] \\
& +C\left(\left\|\sqrt{\rho} w_{t}\right\|_{L^{2}}^{2}+\left\|B_{t}\right\|_{L^{2}}^{2}\right) \\
& \times\left[\|v\|_{L^{\infty}}^{2}+\|\nabla v\|_{L^{\infty}}+\|\nabla w\|_{L^{2}}\left\|\nabla^{2} w\right\|_{L^{2}}+\|\nabla B\|_{L^{2}}\left\|\nabla^{2} B\right\|_{L^{2}}\right] \\
& +C\left[\|v\|_{L^{\infty}}^{4}+\|\nabla v\|_{L^{6}}^{2}+\|\Delta w\|_{L^{2}}^{2}+\left\|\Delta v-\nabla \Pi_{v}\right\|_{L^{4}}^{2}+\left\|\nabla^{2} v\right\|_{L^{6}}^{2}\right] \\
\stackrel{\operatorname{def}}{=} & C\left(\left\|\sqrt{\rho} w_{t}\right\|_{L^{2}}+\left\|B_{t}\right\|_{L^{2}}\right) f_{3}(t)+C\left(\left\|\sqrt{\rho} w_{t}\right\|_{L^{2}}^{2}+\left\|B_{t}\right\|_{L^{2}}^{2}\right) f_{1}(t)+f_{2}(t) \\
\leq & C\left(f_{3}(t)+f_{1}(t)\right)\left(\left\|\sqrt{\rho} w_{t}\right\|_{L^{2}}^{2}+\left\|B_{t}\right\|_{L^{2}}^{2}\right)+f_{2}(t)+f_{3}(t) .
\end{aligned}
$$

We use

$$
\begin{aligned}
& f_{1}(t) \stackrel{\text { def }}{=}\|v\|_{L^{\infty}}^{2}+\|\nabla v\|_{L^{\infty}}+\|\nabla w\|_{L^{2}}\left\|\nabla^{2} w\right\|_{L^{2}}+\|\nabla B\|_{L^{2}}\left\|\nabla^{2} B\right\|_{L^{2}}, \\
& f_{2}(t) \stackrel{\text { def }}{=}\|v\|_{L^{\infty}}^{4}+\|\nabla v\|_{L^{6}}^{2}+\|\Delta w\|_{L^{2}}^{2}+\left\|\Delta v-\nabla \Pi_{v}\right\|_{L^{4}}^{2}+\left\|\nabla^{2} v\right\|_{L^{6}}^{2}, \\
& f_{3}(t) \stackrel{\text { def }}{=}\left\|\nabla\left(\Delta v-\nabla \Pi_{v}\right)\right\|_{L^{4}}+\left\|\partial_{t}\left(\Delta v-\nabla \Pi_{v}\right)\right\|_{L^{2}}+\left\|v_{t}\right\|_{L^{\infty}}+\left\|\nabla v_{t}\right\|_{L^{4}}
\end{aligned}
$$

Applying Gronwall's inequality to (3.23) yields for $t \in\left(t_{1}, T^{*}\right)$,

$$
\begin{aligned}
& \left\|\sqrt{\rho} w_{t}(t)\right\|_{L^{2}}^{2}+\left\|B_{t}(t)\right\|_{L^{2}}^{2}+\int_{t_{1}}^{t}\left\|\nabla w_{t}\left(t^{\prime}\right)\right\|_{L^{2}}^{2}+\left\|\nabla B_{t}\left(t^{\prime}\right)\right\|_{L^{2}}^{2} d t^{\prime} \\
& \leq C \exp \left\{C \int_{t_{1}}^{t}\left(f_{1}\left(t^{\prime}\right)+f_{3}\left(t^{\prime}\right)\right) d t^{\prime}\right\} \\
& \quad \times\left(\left\|\left(\sqrt{\rho} w_{t}\right)\left(t_{1}\right)\right\|_{L^{2}}^{2}+\left\|B_{t}\left(t_{1}\right)\right\|_{L^{2}}^{2}+\int_{t_{1}}^{t}\left(f_{2}\left(t^{\prime}\right)+f_{3}\left(t^{\prime}\right)\right) d t^{\prime}\right) .
\end{aligned}
$$

However, notice that $\dot{B}_{2,1}^{3 / 2} \hookrightarrow L^{\infty}, \dot{B}_{2,1}^{1} \hookrightarrow L^{6}, \dot{B}_{2,1}^{3 / 4} \hookrightarrow L^{4}$, and we deduce from (3.3), (3.4), (3.5), (3.7), and (3.10) that

$$
\int_{t_{1}}^{t}\left(f_{1}\left(t^{\prime}\right)+f_{2}\left(t^{\prime}\right)+f_{3}\left(t^{\prime}\right)\right) d t^{\prime} \leq C
$$


with $C$ being independent of $t$. Taking the $L^{2}$ inner product of the $w, B$ equation of (1.8) with $w_{t}, B_{t}$ at $t=t_{1}$ and using (3.2) give rise to

$$
\begin{aligned}
\left\|\left(\sqrt{\rho} w_{t}\right)\left(t_{1}\right)\right\|_{L^{2}} & \leq C\left(\left\|a\left(t_{1}\right)\right\|_{L^{2}}\left\|\left(\partial_{t} v+v \cdot \nabla v\right)\left(t_{1}\right)\right\|_{\dot{B}_{2,1}^{3 / 2}}+\left\|B \cdot \nabla B\left(t_{1}\right)\right\|_{L^{2}}\right) \\
& \leq C\left(\left\|v\left(t_{1}\right)\right\|_{\dot{B}_{2,1}^{7 / 2}}+\left\|v\left(t_{1}\right)\right\|_{\dot{B}_{2,1}^{3 / 2}}\left\|v\left(t_{1}\right)\right\|_{\dot{B}_{2,1}^{5 / 2}}+\left\|B\left(t_{1}\right)\right\|_{\dot{B}_{2,1}^{1 / 2}}^{\frac{5}{4}}\left\|B\left(t_{1}\right)\right\|_{\dot{B}_{2,1}^{5 / 2}}^{\frac{3}{4}}\right) \\
& \leq C \\
\left\|B_{t}\left(t_{1}\right)\right\|_{L^{2}} \leq & \left(C\left\|v \cdot \nabla B\left(t_{1}\right)\right\|_{L^{2}}+\left\|\Delta B\left(t_{1}\right)\right\|_{L^{2}}+\left\|B \cdot \nabla v\left(t_{1}\right)\right\|_{L^{2}}\right) \\
\leq & C\left(\left\|v\left(t_{1}\right)\right\|_{\dot{B}_{2,1}^{1 / 2}}\left\|B\left(t_{1}\right)\right\|_{\dot{B}_{2,1}^{1 / 2}}^{\frac{1}{4}}\left\|B\left(t_{1}\right)\right\|_{\dot{B}_{2,1}^{5 / 2}}^{\frac{3}{4}}\right. \\
& \left.+\left\|B\left(t_{1}\right)\right\|_{\dot{B}_{2,1}^{1 / 2}}\left\|v\left(t_{1}\right)\right\|_{\dot{B}_{2,1}^{1 / 2}}^{\frac{1}{4}}\left\|v\left(t_{1}\right)\right\|_{\dot{B}_{2,1}^{5 / 2}}^{\frac{3}{4}}+\left\|B\left(t_{1}\right)\right\|_{\dot{B}_{2,1}^{2}}\right) \\
\leq & C .
\end{aligned}
$$

As a consequence, we deduce from (3.25) that

$$
\sup _{t \in\left[t_{1}, T^{*}\right)}\left(\left\|\sqrt{\rho} w_{t}(t)\right\|_{L^{2}}^{2}+\left\|B_{t}(t)\right\|_{L^{2}}^{2}\right)+\int_{t_{1}}^{t}\left\|\nabla w_{t}\left(t^{\prime}\right)\right\|_{L^{2}}^{2}+\left\|\nabla B_{t}\left(t^{\prime}\right)\right\|_{L^{2}}^{2} d t^{\prime} \leq C .
$$

Step 2. The estimate of $\left(\nabla^{2} w, \nabla^{2} B\right)$.

We first observe from the $w$ and $B$ equation of (1.8) that

$$
\begin{aligned}
\left\|\nabla^{2} w\right\|_{L^{2}}+\left\|\nabla \Pi_{w}\right\|_{L^{2}} \leq \varepsilon\left(\left\|\nabla^{2} w\right\|_{L^{2}}+\left\|\nabla^{2} B\right\|_{L^{2}}\right)+C\left\{\left\|\sqrt{\rho} w_{t}\right\|_{L^{2}}+\|\nabla w\|_{L^{2}}^{3}+\|\nabla B\|_{L^{2}}^{3}\right. \\
\left.+\|v\|_{L^{\infty}}\|\nabla w\|_{L^{2}}+\|\nabla v\|_{L^{\infty}}\|w\|_{L^{2}}+\|v\|_{\dot{B}_{2,1}^{7 / 2}}+\|v\|_{\dot{B}_{2,1}^{3 / 2}}^{3 / 2}\|\|_{\dot{B}_{2,1}^{5 / 2}}\right\} \\
\left\|\nabla^{2} B\right\|_{L^{2}} \leq\left\|B_{t}\right\|_{L^{2}}+\|(v+w) \cdot \nabla B\|_{L^{2}}+\|\nabla(v+w) \cdot B\|_{L^{2}} \\
\leq \varepsilon\left(\left\|\nabla^{2} w\right\|_{L^{2}}+\left\|\nabla^{2} w\right\|_{L^{2}}\right)+C\left\{\|\nabla w\|_{L^{2}}^{2}\|\nabla B\|_{L^{2}}+\|\nabla B\|_{L^{2}}^{2}\|\nabla w\|_{L^{2}}\right. \\
\left.+\left\|B_{t}\right\|_{L^{2}}+\|v\|_{L^{\infty}}\|\nabla B\|_{L^{2}}+\|\nabla v\|_{L^{\infty}}\|B\|_{L^{2}}\right\}
\end{aligned}
$$

which along with (3.4), (3.5), (3.7), (3.10), and (3.26) ensures that

$$
\sup _{t \in\left[t_{1}, T^{*}\right)}\left(\left\|\nabla^{2} w(t)\right\|_{L^{2}}+\left\|\nabla^{2} B(t)\right\|_{L^{2}}+\left\|\nabla \Pi_{w}(t)\right\|_{L^{2}}\right) \leq C .
$$

On the other hand, let $(v, q)$ solve

$$
-\Delta v+\nabla q=f, \quad \operatorname{div} v=0 .
$$

Then one has $\nabla q=-\nabla(-\Delta)^{-1} \operatorname{div} f$ and, for any $r \in(1, \infty)$,

$\|\nabla q\|_{L^{r}} \leq C\|f\|_{L^{r}} \quad$ and $\quad\|\Delta v\|_{L^{r}} \leq C\|f\|_{L^{r}}$

from this and the $w$ equation of (1.8), we infer

$$
\begin{aligned}
\left\|\nabla^{2} w\right\|_{L^{6}}+\left\|\nabla \Pi_{w}\right\|_{L^{6}} \leq & C\left(\left\|w_{t}\right\|_{L^{6}}+\|w \cdot \nabla w\|_{L^{6}}+\|v \cdot \nabla w\|_{L^{6}}+\|w \cdot \nabla v\|_{L^{6}}\right. \\
& \left.+\|(1-\rho)\|_{L^{6}}\left\|\partial_{t} v+v \cdot \nabla v\right\|_{L^{\infty}}+\|B \cdot \nabla B\|_{L^{6}}\right),
\end{aligned}
$$


which along with (3.10) implies

$$
\begin{aligned}
& \left\|\nabla^{2} w\right\|_{L^{6}}+\left\|\nabla \Pi_{w}\right\|_{L^{6}} \\
& \leq C\left(\left\|\nabla w_{t}\right\|_{L^{2}}+\|\nabla w\|_{L^{2}}\left\|\nabla^{2} w\right\|_{L^{2}}^{\frac{1}{2}}\left\|\nabla^{2} w\right\|_{L^{6}}^{\frac{1}{2}}+\left\|\nabla^{2} w\right\|_{L^{2}}\|v\|_{L^{\infty}}+\|\nabla w\|_{L^{2}}\|\nabla v\|_{L^{\infty}}\right. \\
& \left.\quad+\|v\|_{\dot{B}_{2,1}^{7 / 2}}+\|v\|_{\dot{B}_{2,1}^{3 / 2}}\|v\|_{\dot{B}_{2,1}^{5 / 2}}+\|\nabla B\|_{L^{2}}\left\|\nabla^{2} B\right\|_{L^{2}}^{\frac{1}{2}}\left\|\nabla^{2} B\right\|_{L^{6}}^{\frac{1}{2}}\right) \\
& \leq \varepsilon\left(\left\|\nabla^{2} w\right\|_{L^{6}}+\left\|\nabla^{2} B\right\|_{L^{6}}\right)+C\left(\left\|\nabla w_{t}\right\|_{L^{2}}+\left\|\nabla^{2} w\right\|_{L^{2}}+\left\|\nabla^{2} B\right\|_{L^{2}}+\|v\|_{\dot{B}_{2,1}^{7 / 2}}+\|v\|_{\dot{B}_{2,1}^{5 / 2}}\right) .
\end{aligned}
$$

On the other hand, following the $B$ equation of (1.8), we infer

$$
\left\|\nabla^{2} B\right\|_{L^{6}} \leq \varepsilon\left(\left\|\nabla^{2} w\right\|_{L^{6}}+\left\|\nabla^{2} B\right\|_{L^{6}}\right)+C\left(\left\|\nabla B_{t}\right\|_{L^{2}}+\left\|\nabla^{2} w\right\|_{L^{2}}+\left\|\nabla^{2} B\right\|_{L^{2}}+\|v\|_{\dot{B}_{2,1}^{5 / 2}}\right) .
$$

Therefore, we get

$$
\begin{aligned}
& \left\|\nabla^{2} w\right\|_{L^{6}}+\left\|\nabla^{2} B\right\|_{L^{6}}+\left\|\nabla \Pi_{w}\right\|_{L^{6}} \\
& \quad \leq C\left(\left\|\nabla w_{t}\right\|_{L^{2}}+\left\|\nabla B_{t}\right\|_{L^{2}}+\left\|\nabla^{2} w\right\|_{L^{2}}+\left\|\nabla^{2} B\right\|_{L^{2}}+\|v\|_{\dot{B}_{2,1}^{7 / 2}}+\|v\|_{\dot{B}_{2,1}^{5 / 2}}\right) .
\end{aligned}
$$

Therefore thanks to (3.4), (3.26), and (3.10), we obtain

$$
\begin{aligned}
& \left\|\nabla^{2} w\right\|_{L^{2}\left(\left[t_{1}, t\right] ; L^{6}\right)}^{2}+\left\|\nabla^{2} B\right\|_{L^{2}\left(\left[t_{1}, t\right] ; L^{6}\right)}^{2}+\left\|\nabla \Pi_{w}\right\|_{L^{2}\left(\left[t_{1}, t\right] ; L^{6}\right)}^{2} \\
& \leq C\left\{\left\|\nabla w_{t}\right\|_{L^{2}\left(\left[t_{1}, t\right] ; L^{2}\right)}^{2}+\left\|\nabla B_{t}\right\|_{L^{2}\left(\left[t_{1}, t\right] ; L^{2}\right)}^{2}+\left\|\nabla^{2} w\right\|_{L^{2}\left(\left[t_{1}, t\right] ; L^{2}\right)}^{2}+\left\|\nabla^{2} B\right\|_{L^{2}\left(\left[t_{1}, t\right] ; L^{2}\right)}^{2}\right. \\
& \left.\quad+\|v\|_{L^{2}\left(\left[t_{1}, t\right] ; \dot{B}_{2,1}^{7 / 2}\right)}^{2}+\|v\|_{L^{2}\left(\left[t_{1}, t\right] ; \dot{B}_{2,1}^{5 / 2}\right)}^{2}\right\} \leq C .
\end{aligned}
$$

This completes the proof of the lemma.

\subsection{Proof of Theorem 1.1}

We first rewrite the momentum equation in (1.3) as

$$
\partial_{t} u+u \cdot \nabla u-\Delta u+\nabla \Pi=(1-\rho)\left(\partial_{t} u+u \cdot \nabla u\right)+B \cdot \nabla B .
$$

Then it follows from the classical theory of the homogeneous Navier-Stokes equations (see [15] for instance) that with $t \in\left[t_{1}, T^{*}\right)$,

$$
\begin{aligned}
& \|u\|_{\tilde{L}^{\infty}\left(\left[t_{1}, t\right] ; \dot{B}_{2,1}^{1 / 2}\right)}+\|u\|_{L^{1}\left(\left[t_{1}, t\right] ; \dot{B}_{2,1}^{5 / 2}\right)}+\|\nabla \Pi\|_{L^{1}\left(\left[t_{1}, t\right] ; \dot{B}_{2,1}^{1 / 2}\right)} \\
& \leq C\left\|u\left(t_{1}\right)\right\|_{\dot{B}_{2,1}^{1 / 2}}+\int_{t_{1}}^{t}\|\nabla u\|_{L^{\infty}}\|u\|_{\dot{B}_{2,1}^{1 / 2}} d t^{\prime}+\left\|(1-\rho)\left(\partial_{t} u+u \cdot \nabla u\right)\right\|_{L^{1}\left(\left[t_{1}, t\right] ; \dot{B}_{2,1}^{1 / 2}\right)} \\
& \quad+\|B \cdot \nabla B\|_{L^{1}\left(\left[t_{1}, t\right] ; \dot{B}_{2,1}^{1 / 2}\right.} .
\end{aligned}
$$

Applying the product law in Besov spaces gives

$$
\left\|(1-\rho)\left(\partial_{t} u+u \cdot \nabla u\right)\right\|_{L^{1}\left(\left[t_{1}, t\right] ; \dot{B}_{2,1}^{1 / 2}\right)} \leq C\|1-\rho\|_{L^{\infty}\left(\left[t_{1}, t\right] \dot{B}_{2,1}^{3 / 2}\right.}\left\|\partial_{t} u+u \cdot \nabla u\right\|_{L^{1}\left(\left[t_{1}, t\right] \dot{B}_{2,1}^{1 / 2}\right.} \cdot
$$


Yet thanks to Lemma 2.1 and (3.18), one has

$$
\begin{aligned}
& \left\|\partial_{t} u\right\|_{L^{1}\left(\left[t_{1}, t\right] ; \dot{B}_{2,1}^{1 / 2}\right)} \leq C t^{1 / 2}\left\|\partial_{t} w\right\|_{L^{2}\left(\left[t_{1}, t\right] ; H^{1}\right)}+\left\|u\left(t_{1}\right)\right\|_{\dot{B}_{2,1}^{1 / 2}} \leq C\left(t^{1 / 2}+1\right), \\
& \|u \cdot \nabla u\|_{L^{1}\left(\left[t_{1}, t\right] ; \dot{B}_{2,1}^{1 / 2}\right)} \leq C \int_{t_{1}}^{t}\left(\|\nabla w\|_{L^{2}}\|\Delta w\|_{L^{2}}+\|v\|_{\dot{B}_{2,1}^{3 / 2}}^{2}\right) d t^{\prime} \leq C, \\
& \|B \cdot \nabla B\|_{L^{1}\left(\left[t_{1}, t\right] ; \dot{B}_{2,1}^{1 / 2}\right)} \leq C \int_{t_{1}}^{t}\|B\|_{\dot{H}^{1}}\|\nabla B\|_{\dot{H}^{1}} d t^{\prime} \leq C \int_{t_{1}}^{t}\|\nabla B\|_{L^{2}}\|\Delta B\|_{L^{2}} d t^{\prime} \leq C .
\end{aligned}
$$

Thanks to Theorem 2.87 in [13] and Proposition 1 in [8], we have

$$
\begin{aligned}
& \|1-\rho\|_{\tilde{L}_{\left(\left[t_{1}, t\right] ; \dot{B}_{2,1}^{3 / 2}\right)}} \\
& \quad \leq C\|a\|_{\tilde{L}^{\infty}\left(\left[t_{1}, t\right] \dot{B}_{2,1}^{3 / 2}\right)} \leq C\left\|a\left(t_{1}\right)\right\|_{\dot{B}_{2,1}^{3 / 2}} \exp \left\{C \int_{t_{1}}^{t}\left\|u\left(t^{\prime}\right)\right\|_{\dot{B}_{6,1}^{3 / 2}} d t^{\prime}\right\} .
\end{aligned}
$$

However, applying Lemma 2.1 leads to

$$
\|u\|_{\dot{B}_{6,1}^{3 / 2}} \leq C\|\nabla w\|_{L^{6}}^{1 / 2}\left\|\nabla^{2} w\right\|_{L^{6}}^{1 / 2}+\|v\|_{\dot{B}_{2,1}^{5 / 2}}
$$

which along with (3.10) and (3.18) implies

$$
\begin{aligned}
& \|\nabla u\|_{L^{1}\left(\left[t_{1}, t\right] ; L^{\infty}\right)}+\|u\|_{L^{1}\left(\left[t_{1}, t\right] ; \dot{B}_{6,1}^{3 / 2}\right)} \\
& \quad \leq C\|u\|_{L^{1}\left(\left[t_{1}, t\right] ; \dot{B}_{6,1}^{3 / 2}\right)} \leq C\left\{\|v\|_{L^{1}\left(\left[t_{1}, t\right] ; \dot{B}_{2,1}^{5 / 2}\right)}+t^{1 / 2}\|\Delta w\|_{L^{2}\left(\left[t_{1}, t\right] ; L^{2}\right)}^{1 / 2}\|\Delta w\|_{L^{2}\left(\left[t_{1}, t\right] ; L^{6}\right)}^{1 / 2}\right\} \\
& \quad \leq C\left(1+t^{1 / 2}\right) .
\end{aligned}
$$

Therefore, we obtain

$$
\left\|(1-\rho)\left(\partial_{t} u+u \cdot \nabla u\right)\right\|_{L^{1}\left(\left[t_{1}, t\right] ; \dot{B}_{2,1}^{1 / 2}\right)} \leq C\left\|a\left(t_{1}\right)\right\|_{\dot{B}_{2,1}^{3 / 2}} \exp \left\{C t^{1 / 2}\right\} .
$$

Then applying Gronwall's inequality to (3.28) gives rise to

$$
\begin{aligned}
& \|u\|_{\widetilde{L}^{\infty}\left(\left[t_{1}, t\right] ; \dot{B}_{2,1}^{1 / 2}\right)}+\|u\|_{L^{1}\left(\left[t_{1}, t\right] ; \dot{B}_{2,1}^{5 / 2}\right)}+\|\nabla \Pi\|_{L^{1}\left(\left[t_{1}, t\right] \dot{B}_{2,1}^{1 / 2}\right)} \\
& \quad \leq C \exp \{C \sqrt{t}\}\left(\left\|u_{0}\right\|_{\dot{B}_{2,1}^{1 / 2}}+\left\|a_{0}\right\|_{B_{2,1}^{3 / 2}}+C\right) .
\end{aligned}
$$

We first observe from the $B$ equation of (1.8) that

$$
\partial_{t} B-\Delta B=u \cdot \nabla B+B \cdot \nabla u .
$$

Similarly, we get

$$
\begin{aligned}
& \|B\|_{\tilde{L}^{\infty}\left(\left[t_{1}, t\right] ; \dot{B}_{2,1}^{1 / 2}\right)}+\|B\|_{L^{1}\left(\left[t_{1}, t\right] ; \dot{B}_{2,1}^{5 / 2}\right)} \\
& \quad \leq C\left\|B\left(t_{1}\right)\right\|_{\dot{B}_{2,1}^{1 / 2}}+\|u \cdot \nabla B\|_{L^{1}\left(\left[t_{1}, t\right] ; \dot{B}_{2,1}^{1 / 2}\right)}+\|B \cdot \nabla u\|_{L^{1}\left(\left[t_{1}, t\right] ; \dot{B}_{2,1}^{1 / 2}\right)} \\
& \quad \leq C\left\|B_{0}\right\|_{\dot{B}_{2,1}^{1 / 2}}+C .
\end{aligned}
$$


From (3.29), (3.30), and (3.31), we infer by a standard argument that $T^{*}=\infty$. Moreover, the global solution thus obtained, $(a, u, B, \Pi)$, satisfies (1.5). This completes the proof of Theorem 1.1.

\section{Competing interests}

The authors declare that they have no competing interests.

\section{Authors' contributions}

All authors contributed equally to the writing of this paper. All authors read and approved the final manuscript.

\section{Author details}

'School of Mathematics, Northwest University, Xi'an, 710069, P.R. China. ${ }^{2}$ School of Mathematics and Information

Science, Henan Polytechnic University, Jiaozuo, 454000, P.R. China.

\section{Acknowledgements}

The research of Xiaolian Ai is partially supported by NSFC11571279, and grants 2014JQ1009 and 14JK1757.

Received: 9 October 2015 Accepted: 4 November 2015 Published online: 25 November 2015

\section{References}

1. Davidson, PA: An Introduction to Magnetohydrodynamics. Cambridge University Press, Cambridge (2001)

2. Desjardins, B, Le Bris, C: Remarks on a nonhomogeneous model of magnetohydrodynamics. Differ. Integral Equ. 11(3), 377-394 (1998)

3. Gerbeau, JF, Le Bris, C: Existence of solution for a density-dependent magnetohydrodynamic equation. Adv. Differ. Equ. 2(3), 427-452 (1997)

4. Abidi, H, Hmidi, T: Résultats d'existence globale pour le systéme de la magnethohydrodynamique inhomogene. Ann. Math. Blaise Pascal 14(1), 103-148 (2007)

5. Chen, Q, Tan, Z, Wang, YJ: Strong solutions to the incompressible magnetohydrodynamic equations. Math. Methods Appl. Sci. 34(1), 94-107 (2011)

6. Huang, XD, Wang, Y: Global strong solution to the 2D nonhomogeneous incompressible MHD system. J. Differ. Equ. 254(2), 511-527 (2013)

7. Gui, G: Global well-posedness of the two-dimensional incompressible magnetohydrodynamic system with variable density and electrical conductivity. J. Funct. Anal. 267(5), 1488-1539 (2015)

8. Abidi, H, Gui, G, Zhang, P: On the wellposedness of three-dimensional inhomogeneous Navier-Stokes equations in the critical spaces. Arch. Ration. Mech. Anal. 204(1), 189-230 (2012)

9. Chemin, JY: Perfect Incompressible Fluids. Oxford University Press, New York (1998)

10. Peetre, J: New Thoughts on Besov Spaces. Duke University Mathematical Series, vol. 1. Duke University Press, Durham (1976)

11. Triebel, H: Theory of Function Spaces. Monographs in Mathematics, vol. 78. Birkhäuser, Basel (1983)

12. Bony, JM: Calcul symbolique et propagation des singularités pour les équations aux dérivées partielles non linéaires. Ann. Sci. Éc. Norm. Super. 14(4), 209-246 (1981)

13. Bahouri, H, Chemin, JY, Danchin, R: Fourier Analysis and Nonlinear Partial Differential Equations. Grundlehren der Mathematischen Wissenschaften, vol. 343. Springer, Berlin (2011)

14. Chemin, JY, Lerner, N: Flot de champs de vecteurs non Lipschitziens et équations de Navier-Stokes. J. Differ. Equ. 121(2), 314-328 (1995)

15. Chemin, JY: Théorémes d'unicité pour le systéme de Navier-Stokes tridimensionnel. J. Anal. Math. 77(1), 27-50 (1999)

16. Li, ZL, Wang, M, Yang, J: On the local wellposedness of three-dimensional MHD system in the critical spaces. Acta Math. Appl. Sinica (Engl. Ser.) 31, 607-622 (2015)

\section{Submit your manuscript to a SpringerOpen ${ }^{\circ}$ journal and benefit from:}

- Convenient online submission

Rigorous peer review

- Immediate publication on acceptance

- Open access: articles freely available online

- High visibility within the field

- Retaining the copyright to your article 